\title{
High-Speed Roll-to-Roll Nanoimprint Lithography on Flexible Plastic Substrates**
}

\author{
By Se Hyun Ahn and L. Jay Guo*
}

The ability of micro- to nanometer-scale patterning on flexible substrates can enable many new applications in the area of photonics and organic electronics. A major roadblock has remained for many practical applications of patterned nanostructures, which is the throughput of nanopattern replication and the associated cost issues. Among the emerging techniques, nanoimprint lithography (NIL) clearly stands out as a promising technology for high-throughput and highresolution nanometer-scale patterning, ${ }^{[1,2]}$ which can achieve resolutions beyond the limitations set by light diffraction or beam scattering that are encountered in other traditional techniques. Developments in this area have enjoyed great momentum in the past decade and numerous applications, such as in $\mathrm{Si}$ electronics, ${ }^{[3,4]}$ organic electronics and photonics, ${ }^{[5,6]}$ magnetics, ${ }^{[7,8]}$ and biology ${ }^{[9-12]}$ have been exploited by many researchers. On the other hand, the current process and throughput in NIL (on the order of a few minutes per wafer) is still far from meeting the demands of many practical applications, especially in photonics, biotechnology, and organic optoelectronics. The concept of roller imprinting has been pursued by previous investigators as a means to improve speed. ${ }^{[13]}$ However, the procedure was to imprint a small piece of Si mold onto a Si substrate, which is not too different from that of conventional NIL except that a rod is used to apply pressure rather than a flat plate. The reverse nanoimprinting ${ }^{[14]}$ or nanotransfer printing methods ${ }^{[15]}$ produce positive-tone polymer or metal patterns, which in principle can also be applied to roll-to-roll printing processes. In addition, Lee et al. proposed a bilayer transfer process from a "rigiflex" mold to a Si wafer, ${ }^{[16]}$ and pointed out that the process can potentially be extended to a roller bilayer transfer process. However, these are yet to be demonstrated.

[*] Prof. L. J. Guo

Department of Electrical Engineering and Computer Science University of Michigan

Ann Arbor, MI 48109 (USA)

E-mail: guo@eecs.umich.edu

S. H. Ahn

Department of Mechanical Engineering

University of Michigan

Ann Arbor, MI 48109 (USA)

[**] This research is supported by NSF (grant No. CMII 0700718), AFOSR (grant No. F064-006-0084), and the University of Michigan Technology Transfer Office (GAP fund). We thank Dr. Jin-Sung Kim for providing the short-period Si mold used in this study.
The motivation of this work is to enable continuous printing of nanostructures on a flexible web with drastically increased throughput, and thereby push the nanometer-scale lithography to an entirely new level. The roll-to-roll nanoimprint lithography (R2RNIL) technology presented in this Communication inherits its high-resolution feature from traditional NIL because it is also based on a mechanical embossing approach, but with a speed of nanopatterning increased by at least one or two orders of magnitude.The R2RNIL process primarily targets large-area patterning of nanostructures. In the conventional approach, embossing a large area requires a very large force. Huge contact areas between the mold surface and the imprinted nanostructures also produce significant adhesion force, making the mold-sample separation without damaging the substrates difficult or even impossible. In thermal NIL, if the mold and substrate are made from materials with different thermal expansion coefficients, such as Si mold and polymer substrate, stress can build up during a thermal cycle of such a magnitude that it can even destroy the Si mold during mold releasing. R2RNIL provides a unique solution to these challenges encountered in the conventional wafer-scale NIL process, because imprinting in R2RNIL proceeds in a narrow region transverse to the web moving direction, thus requiring a much smaller force to replicate the patterns. Also, because the mold used in R2RNIL is in the form of a roller, the mold-sample separation proceeds in a "peeling" fashion, which requires much less force and reduces the probability of defect generation.

True roll-to-roll nanoimprinting has been a challenge to the community because it requires a complete set of solutions to address a number of interrelated material issues. First of all, a special roller mold is required for continuous roll-to-roll imprinting of nanostructures. Molds used in R2RNIL should be flexible enough to wrap onto a roller surface, and they should also have sufficient modulus and strength to imprint other materials. A low surface energy is essential for clean mold release. In addition, the roller mold should be durable under continuous imprinting. In this study, a flexible fluoropolymer, ethylene-tetrafluoroethylene (ETFE), is used as mold material. ETFE has a high modulus (ca. 1.2 GPa) at room temperature but can be softened at elevated temperatures. Therefore, an ETFE mold can be easily replicated (Fig. 1a) from an original Si mold by a thermal NIL process at $220^{\circ} \mathrm{C}^{[3]}$ while the original large-area $\mathrm{Si}$ mold can be fabricated by laser interference lithography. ${ }^{[17,18]}$ Moreover, the exceptional antisticking properties of ETFE (surface 


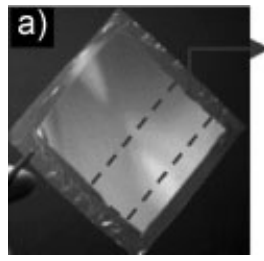

b)

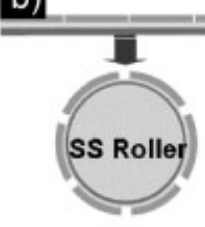

Figure 1. a) A flexible ETFE mold fabricated from an original Si mold. The ETFE mold assembly is prepared as shown in (b) and attached to a $60 \mathrm{~mm}$ diameter stainless-steel roller. A soft cushion layer is placed between the ETFE mold assembly and the roller surface for conformal contact during imprinting.

energy of $15.6 \mathrm{dyn}^{-1}$, cf. poly(dimethylsiloxane) (PDMS) ca. $19.6 \mathrm{dyn} \mathrm{cm}^{-1}$ ) make it easy to demold after imprinting without any mold surface treatment and without deterioration in surface properties over long imprinting cycles. In our experiment, several pieces of ETFE molds of proper size were replicated, wrapped, and fixed to a $60 \mathrm{~mm}$ diameter stainless-steel roller.

For a fast roll-to-roll process, there are several stringent requirements for the resist materials. First, liquid resists having good coating properties are preferred because they can be continuously and uniformly coated onto plastic substrates and be easily imprinted with low pressure. Second, such liquid resists should have low viscosity before curing for fast imprinting. Third, resists used in R2RNIL need to be quickly cured and with minimum shrinkage after curing. Therefore, conventional resist materials dissolved in solvents and requiring additional baking processes are not suitable for the R2RNIL process. Thermoplastic materials that are typically used in conventional NIL are also not adequate for R2RNIL because they require very high pressures and relatively long processing times to complete the imprinting. In our experiment, two types of liquid resist were exploited for R2RNIL application. For thermal R2RNIL, a fast, thermal-curable liquid resist ${ }^{[19]}$ based on a modified PDMS was used. The resist consists of four basic chemical components: a vinyl terminated polydimethylsiloxane polymer, a silyl-hydride $(\mathrm{Si}-\mathrm{H})$ based dimethylsiloxane crosslinker, a platinum catalyst, and an inhibitor. The inhibitor is an unsaturated organic ester that keeps the catalyst inactive until the application of heat, which quickly deactivates the inhibitor and releases the catalyst in its active form. The low-viscosity liquid resist can quickly fill into the cavity features on the mold surface by the web tension and the pressure from the backup rollers. The material can be crosslinked within a few seconds at a temperature of $120^{\circ} \mathrm{C}$ because of the rapid deactivation of the inhibitor. The fast crosslinking ensures high-speed patterning required for R2RNIL

For even higher-speed R2RNIL, we used a UV-curable low viscosity liquid epoxysilicone ${ }^{[20]}$ as imprint resist material. Different from acrylate-based resists often used in UV-assisted NIL process such as step-and flash imprint lithography (S-FIL), ${ }^{[21]}$ epoxysilicone is cured via a cationic curing mechanism, thereby free from the oxygen sensitivity issue when exposed in air. Thus no special vacuum environment is
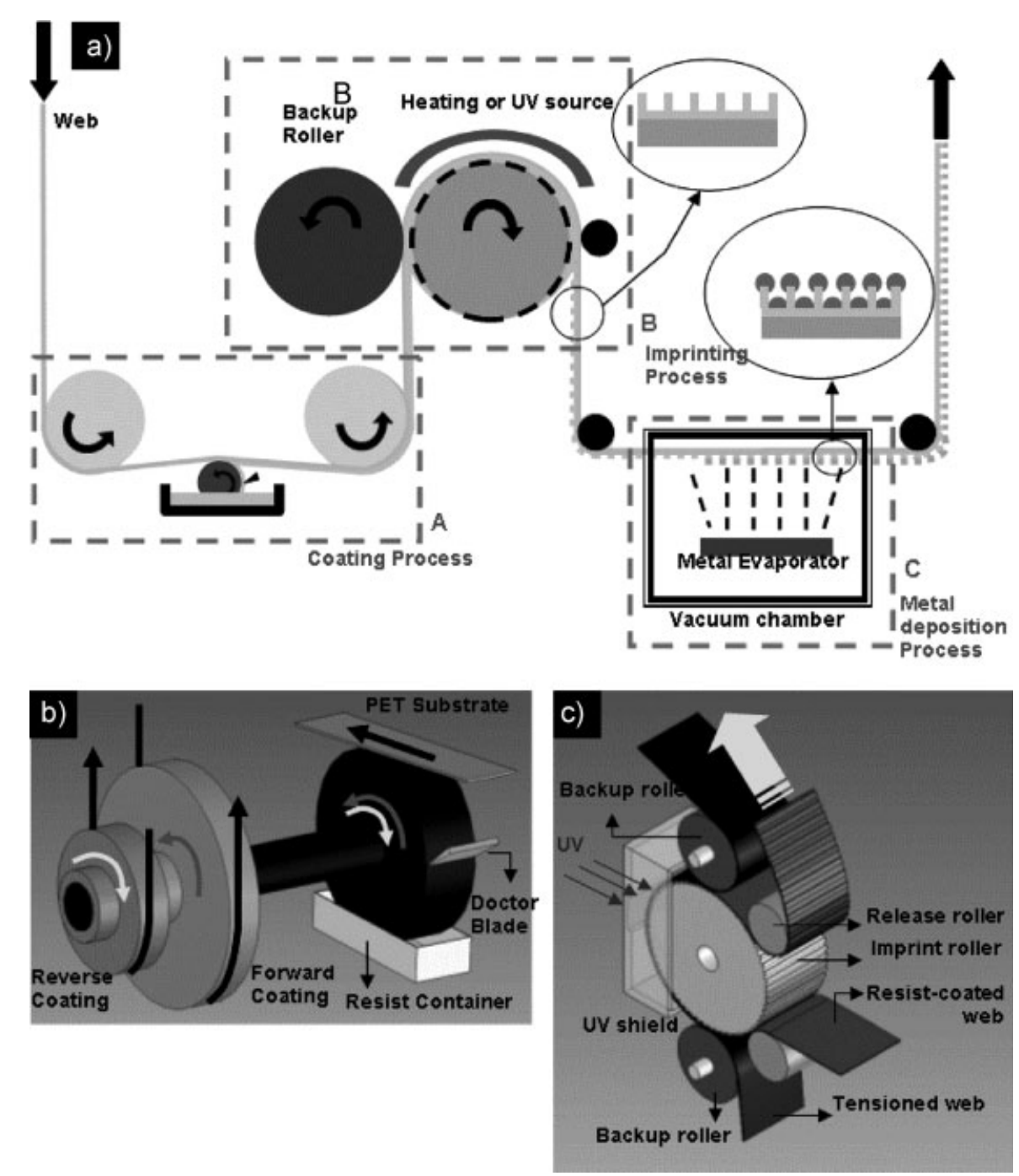

Figure 2. a) Schematic of the R2RNIL process, and the continuous fabrication of a metal wire-grid polarizer as one of its applications (the metal deposition is carried out in a separate evaporator in this study). b) The coating unit and c) the Imprint unit of the R2RNIL apparatus. 


\section{ADVANCED materials}

required, which is convenient for the roll-to-roll process. Furthermore, its very low shrinkage after curing (only a fraction of the acrylate system) allows a faithful pattern replication. Owing to its low viscosity, the resist precursor can be imprinted at low pressures and cured within a second by focused UV light. The low pressure and room temperature imprinting characteristics are advantageous for R2RNIL.

The resist film uniformity on the flexible web and the resist thickness are important from both pattern quality and economic point of view. Too thin a film may cause insufficient filling of resist into certain mold regions or cause film fracture during mold releasing. Too thick a resist not only results in accumulation of resist from successive rolling cycles that hampers further imprinting, but also squeezes out excess materials resulting in resist waste. In our experiment, two types of coating methods, reverse and forward web coating, are used. Reverse coating, in which the web direction is opposite to the rolling direction, provides a uniform coating profile by eliminating film separation. However, such coating tends to be rather thick. Forward coating provides a thinner film layer but a slightly lower surface uniformity. In our R2RNIL system, we can easily select either forward or reverse coating (Fig. 2b), and adjust the speed ratio of the moving web and the coating roller that determines the film thickness. Liquid resist is transferred from a resist-container to the flexible PET substrate by the coating roller and its final thickness is controlled by a doctor blade.

The imprint section is the most important part in the R2RNIL system, because pattern quality mostly depends on the pressure of the imprint roller and curing conditions. Our imprint module is composed of an imprint roller, two backup rollers, a release roller, and a curing section (Fig. 2c). Under the web tension and the pressure from the backup roller, the low-viscosity monomer resist quickly fills into mold cavity. Moreover, the two backup rollers with a spring system also guarantee non-slip motion in the rolling process, which is very important for successful pattern replication. In the next step, the resist precursor is cured either by convection heating (Steinel heat gun) or UV irradiation (Omnicure1000 highpower UV source, EXFO). A box-shaped radiation shield is used to limit the UV exposure only in the curing region, preventing premature curing of the resist before reaching the imprinting zone. Finally, a poly(ethylene terephthalate) (PET) substrate with roller-imprinted nanostructures continuously separates from the roller mold via the release roller.

For easy visualization of the imprinting results, we chose a grating pattern of $700 \mathrm{~nm}$ period in our initial experiment, because a well-replicated grating structure should show strong light diffraction and therefore the pattern quality can be easily examined by the eye. We first performed continuous nanoimprinting on a flexible plastic substrate by using a thermally curable liquid resist. A $200 \mathrm{~mm}$ long, $300 \mathrm{~nm}$ linewidth, and $700 \mathrm{~nm}$ period grating imprinted by using the thermally cured PDMS on a PET substrate is shown in Figure $3 \mathrm{a}$ and $\mathrm{b}$. Curing of PDMS liquid resist was done by convection heating using a heat gun. Next, we experimented with UV-curable epoxysilicone liquid resist. Figure 3c-e shows UV R2RNIL results of a $570 \mathrm{~mm}$ long (width $10 \mathrm{~mm}$ ), $700 \mathrm{~nm}$ period grating structure created on PET substrate. Scanning electron microscopy (SEM) shows that the UV-cured
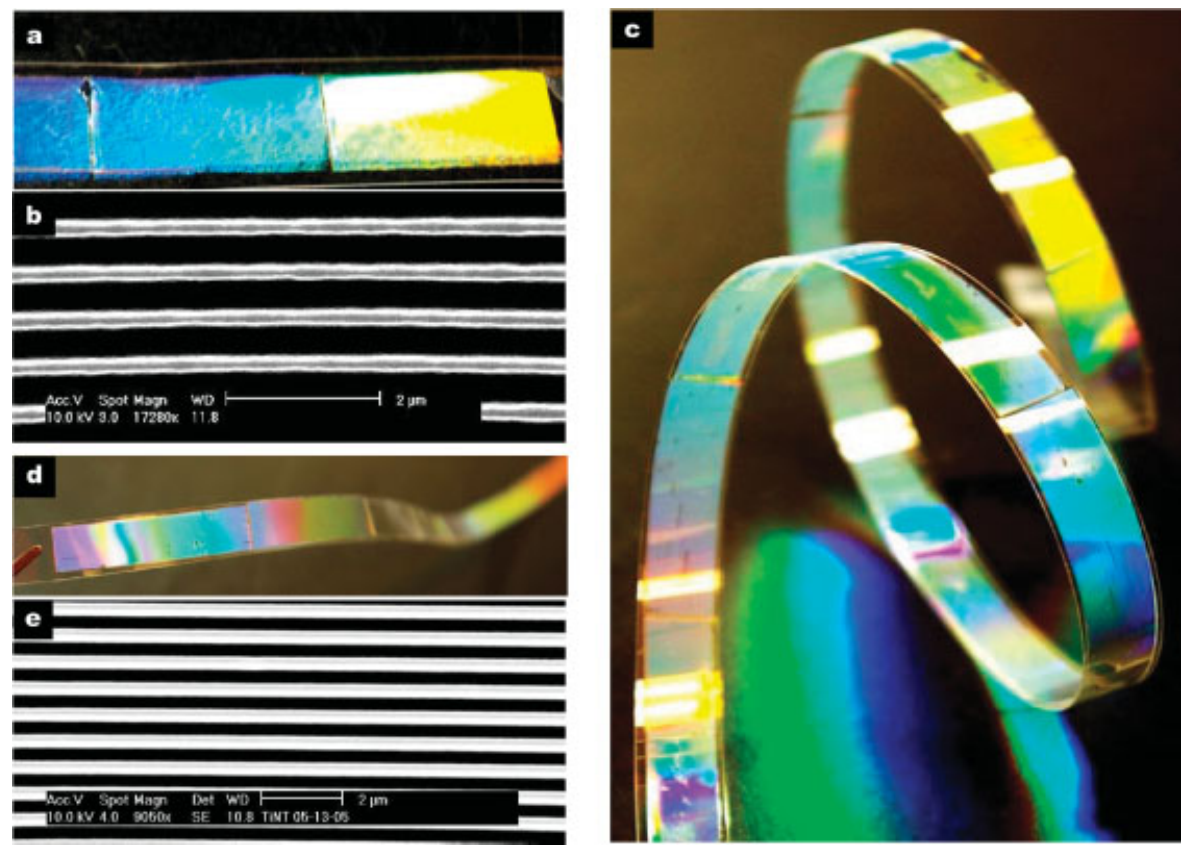

Figure 3. Thermal R2RNIL results: a) Photograph of a $700 \mathrm{~nm}$ period, $300 \mathrm{~nm}$ line width PDMS grating pattern imprinted on PET strip by using the thermal R2RNIL and b) the SEM micrograph of the replicated grating structure. UV R2RNIL results: c,d) Photographs of $700 \mathrm{~nm}$ period, $300 \mathrm{~nm}$ line width epoxysilicone grating pattern imprinted on PET strip by the UV R2RNIL, showing bright light diffraction, and e) SEM image of the replicated grating structure. Total length is $570 \mathrm{~mm}$. 

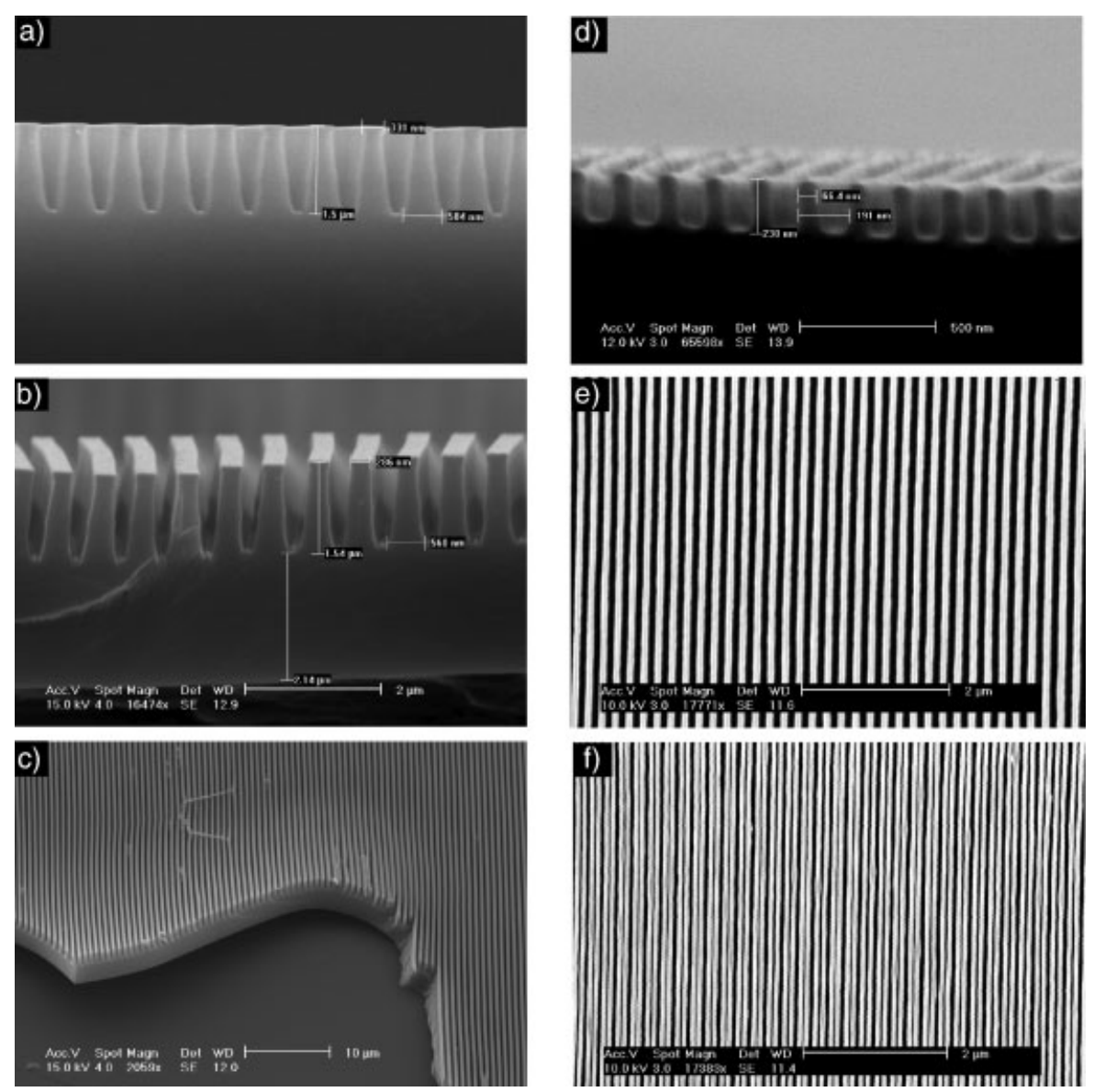

Figure 4. a) The original Si mold. b,c) Epoxysilicone gratings replicated from the ETFE mold. d,e) SEM pictures of $200 \mathrm{~nm}$ period, $70 \mathrm{~nm}$ line width epoxysilicone pattern, and f) a $100 \mathrm{~nm}$ period, $70 \mathrm{~nm}$ line width epoxysilicone pattern fabricated by UV R2RNIL.

epoxysilicone resist pattern has a higher quality than the thermally cured PDMS, which can be attributed to the lower viscosity of the epoxysilicone material that facilitates the fast filling of the mold cavity. The printing speed can be adjusted depending on the period of the grating pattern and its aspect ratio. The fast curing of the resist material allowed us to achieve a web speed of ca. $1 \mathrm{~m} \mathrm{~min}^{-1}$.

High-aspect-ratio $(\mathrm{AR}=5.4: 1)$ grating structures with very sharp pattern definition fabricated by R2RNIL are shown in Figure $4 \mathrm{~b}$ and c. Faithfully replicated epoxysilicone pattern should have the same geometry as in the original Si mold (Fig. 4a) because the ETFE mold, replicated from the Si mold, has the exact inverse patterns of the Si mold. Comparing the grating structure on the original Si mold (Fig. 4a) and the imprinted epoxysilicone pattern (Fig. 4c), we observe excellent pattern replication even for the very fine details at the bottom of the grating trenches. The residual layer thickness in this result is about $2 \mu \mathrm{m}$, but can be controlled through the web tension and backup roller pressure. Even though the ETFE mold has good antisticking properties, such a high-aspect-ratio imprinted structure tends to show significant sticking to the
ETFE mold owing to the much larger contact area with the grating sidewalls on the mold. To achieve successful pattern transfer, we performed oxygen plasma treatment followed by thermal deposition of Silquest A187 adhesion promoter (GE Advance Materials) on the PET substrate before imprinting, which improves the adhesion of the resist pattern to the PET substrate. In addition, a few drops of fluorosurfactant were added to the epoxysilicone resist to further reduce the adhesion between the imprinted pattern and the ETFE mold surface.

Continuous roll-to-roll imprinting of thinner and denser grating structures is more challenging because such patterns are mechanically fragile, and tend to collapse during demolding if the trench is very small. This requires the cured resist to have sufficient modulus and yield strength. Good adhesion of the resist to the substrate is also very important for such denser structures, which was achieved by using the aforementioned adhesion promoter. Figure $4 \mathrm{~d}$ and e show $200 \mathrm{~nm}$ period, $70 \mathrm{~nm}$ linewidth epoxysilicone patterns by the UV R2RNIL process. A SEM image of $100 \mathrm{~nm}$ period grating structure is also replicated successfully and shown in Figure 4f.

To demonstrate an application of the R2RNIL process, we fabricated a metal wire-grid polarizer. By depositing a thin metal (Al) layer over the imprinted grating structures, a high-efficiency polarizer in the form of bilayer metal wire grating can be achieved. ${ }^{[22,23]}$ In our initial experiment, a $200 \mathrm{~nm}$ and $100 \mathrm{~nm}$ period grating pattern (Fig. 4d-f) were prepared by the R2RNIL process and various thicknesses of aluminum were thermally deposited on top of the grating as well as at the bottom of the trench (Fig. 5a and b). To quantify the polarization effect, the spectral transmittance was measured using UV-vis spectrometry. Figure 5c shows the transmittance of the TM, TE polarized light through the fabricated metal wire-grid polarizer. The best polarizer result was obtained from the $100 \mathrm{~nm}$ period grating with a $50 \mathrm{~nm} \mathrm{Al}$ layer, with a transmittance of ca. $30 \%$ at $800 \mathrm{~nm}$ wavelength, an extinction ratio (transmittance of $\mathrm{TM} /$ transmittance of $\mathrm{TE}$ ) over 2000 at $700-800 \mathrm{~nm}$ wavelength, and a 2500 maximum extinction ratio at $770 \mathrm{~nm}$. We would like to point out that in these preliminary experiments, many parameters such as grating period, line width, and metal thickness have not yet been optimized. 

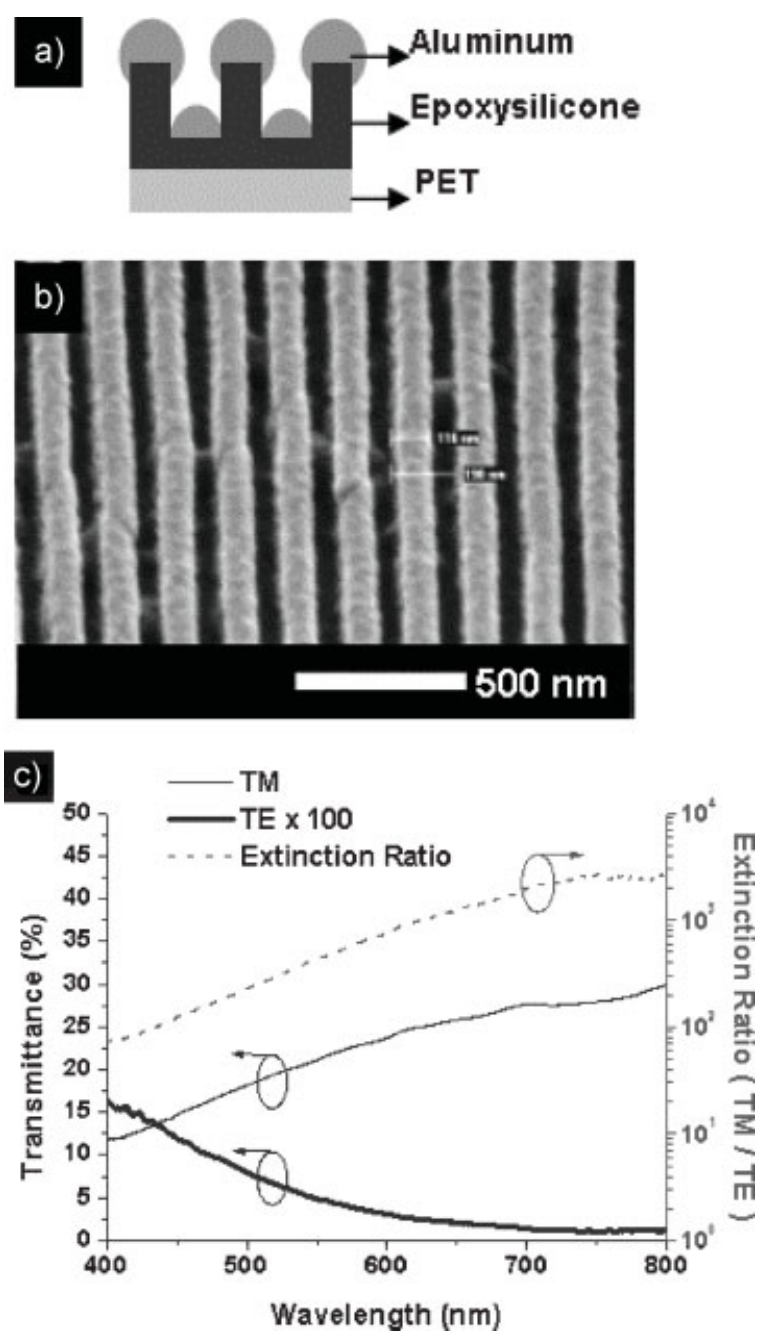

Figure 5. a) Schematic of the metal wire-grid polarizer by depositing metal on top of the roller imprinted polymer grating. b) SEM image of a $200 \mathrm{~nm}$ period grating with $50 \mathrm{~nm}$ Al on top. c) Spectral transmittance (TM, TE mode) and extinction ratio (TM/TE) of a metal wire-grid polarizer fabricated by R2RNIL.

In summary, we have demonstrated a true R2RNIL process in which polymer patterns down to $70 \mathrm{~nm}$ feature size were continuously imprinted on a flexible web. This result represents a drastic increase in the process throughput, which addresses one of the bottlenecks in nanopatterning for many practical applications.

\section{Experimental}

Fabrication of Si Mold: $\mathrm{SiO}_{2}$ of $200 \mathrm{~nm}$ thickness was thermally grown on a $\mathrm{Si}$ wafer, and then chrome $(\mathrm{Cr})$ of $10 \mathrm{~nm}$ thickness was deposited onto the $\mathrm{SiO}_{2}$ layer by using a radiofrequency (RF) sputter. The Cr layer was used for the improvement of adhesion properties between the photoresist (PR) and the $\mathrm{SiO}_{2}$ layer as well as for an etch mask to the $\mathrm{SiO}_{2}$ layer. PR of $90 \mathrm{~nm}$ thickness was spin-coated onto the $\mathrm{Cr}$ layer, and laser interference lithography using a $325 \mathrm{~nm}$ wavelength

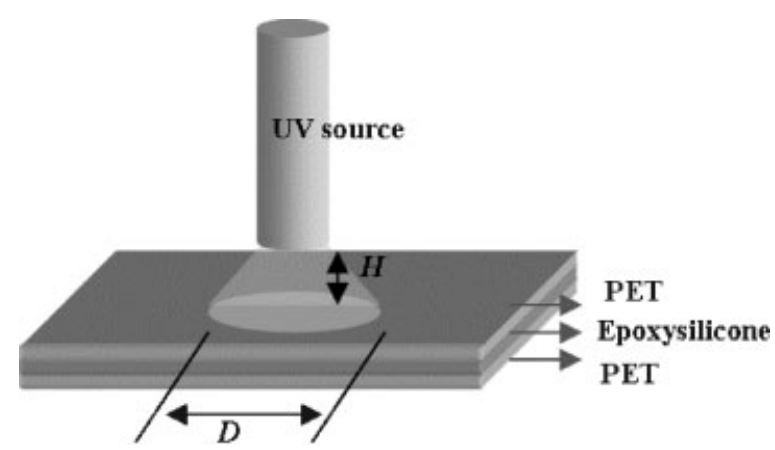

Figure 6. Schematic of UV curing test of epoxysilicone. UV curable epoxysilicone resist is sandwiched by two sheets of PET film, as in the actual R2RNIL process. $H$ is the distance between the light source and the sample, and $D$ is the diameter of fully solidified area after curing.

He-Cd laser was performed to form a grating pattern of $200 \mathrm{~nm}$ pitch on the PR. The $\mathrm{Cr}$ layer was etched with $\mathrm{Cl}_{2}$ and $\mathrm{O}_{2} \mathrm{RIE}$, and the $\mathrm{SiO}_{2}$ layer with $\mathrm{CHF}_{3}$ RIE. After etching the $\mathrm{SiO}_{2}$, the $\mathrm{Cr}$ mask was removed by $\mathrm{Cr}$ wet etchant. The fabricated stamp was pretreated by an antisticking layer $(1 \mathrm{H}, 1 \mathrm{H}, 2 \mathrm{H}, 2 \mathrm{H}$-Perfluorodecyl trichlorosilane) to prevent the stamp from adhering to the imprint pattern for the demolding step.

Fabrication of ETFE Mold: A $200 \mu \mathrm{m}$ thick ETFE film (SAINTGOBAIN) was sandwiched between a Si mold containing grating structures and a Si substrate, and pressed by a pressure machine with a temperature controller. A temperature of $220^{\circ} \mathrm{C}$ and an imprint pressure of $2 \mathrm{MPa}$ were used. After $5 \mathrm{~min}$ hot-embossing, the sample was cooled to ambient temperature while keeping the pressure at $2 \mathrm{MPa}$. Then, the ETFE film was released from the Si mold by manual peeling.

Surface Treatment of PET Substrate: To improve the adhesion of the PET substrate with the resist pattern, especially for the high-aspect-ratio $(>5)$ or small-pitch $(200 \mathrm{~nm} / 100 \mathrm{~nm}$ period) grating structures, the PET substrate was pretreated by Oxygen plasma $\left(100 \mathrm{~W}, 250 \mathrm{mTorr}\left(1\right.\right.$ Torr $\left.\left.=1.333 \times 10^{2} \mathrm{~Pa}\right), 10 \mathrm{~min}\right)$ followed by thermal deposition of adhesion promoter, Silquest187 (GE Advance Materials, $\left.100^{\circ} \mathrm{C}, 15 \mathrm{~min}\right)$.

Roll-to-Roll Imprint: A flexible ETFE mold was wrapped on a stainless steel imprint roller (diameter $=60 \mathrm{~mm}$, width $=10 \mathrm{~mm}$ ) using double-sided adhesion tape. A $1 \mathrm{~mm}$ thick rubber cushion layer was placed between the roller and the ETFE mold to ensure adequate conformal contact. Liquid resist material was continuously coated on the flexible PET substrate in the coating module. The amount of resist material was adjusted by the doctor blade fixed in the two-DOF (linear, tilting) stage. The PDMS resist precursor was cured by convention heating (hot-air gun, Steinel) and epoxysilicone resist by UV exposure $\left(7.2 \mathrm{~W} \mathrm{~cm}^{-2}\right.$, EXFO Inc.). For the UV curing, the light intensity depended on the opening ratio of iris and the distance from the source.

Table 1. Diameter of the fully solidified area after curing $(D)$ of epoxysilicone as a function of the curing time $(t)$, the iris opening ratio, and the distance from UV light source $(H)$.

\begin{tabular}{lrccc}
\hline Opening Ratio[a] & $H[\mathrm{~b}]$ & $t=1 \mathrm{~s}$ & $t=0.5 \mathrm{~s}$ & $t=0.2 \mathrm{~s}$ \\
\hline $90 \%$ & $15 \mathrm{~mm}$ & $D=20 \mathrm{~mm}$ & $D=15 \mathrm{~mm}$ & $D=15 \mathrm{~mm}$ \\
& $5 \mathrm{~mm}$ & $D=12 \mathrm{~mm}$ & $D=10 \mathrm{~mm}$ & $D=10 \mathrm{~mm}$ \\
$40 \%$ & $15 \mathrm{~mm}$ & $D=15 \mathrm{~mm}$ & $D=15 \mathrm{~mm}$ & $D=12 \mathrm{~mm}$ \\
& $5 \mathrm{~mm}$ & $D=10 \mathrm{~mm}$ & $D=10 \mathrm{~mm}$ & $D=10 \mathrm{~mm}$ \\
\hline
\end{tabular}

[a][a]/ris opening ratio. [b][b]Distance between UV source and sample. 
To determine the curing time and the cured area in relation to the iris opening ratio and distance from UV source, an epoxysilicone resist (10\% crosslinker formulation) [20] sandwiched by two PET films was cured by UV irradiation (Fig. 6). The test results are shown in Table 1. When the gap was $5 \mathrm{~mm}, 10 \mathrm{~mm}$ diameter area of epoxysilicone was cured within $0.2 \mathrm{~s}$ for both $90 \%$ and $40 \%$ opening. In the actual R2RNIL process, the resist was cured by the UV light source placed $15 \mathrm{~mm}$ away and at $40 \%$ opening. The web speed $\left(1.3-23.5 \mathrm{~mm} \mathrm{~s}^{-1}\right)$ was controlled by an ac motor controller.

Al Deposition for the Wire-Grid Polarizer: Al film of various thicknesses $(50 \mathrm{~nm}, 70 \mathrm{~nm}, 100 \mathrm{~nm})$ was thermally deposited on the grating structures in a separate evaporator.

Received: October 23, 2007 Published online: April 24, 2008

[1] S. Y. Chou, P. R. Krauss, P. J. Renstrom, Appl. Phys. Lett. 1995, 67, 3114.

[2] S. Y. Chou, P. R. Krauss, P. J. Renstrom, Science 1996, $272,85$.

[3] L. J. Guo, Adv. Mater. 2007, 19, 495.

[4] W. Zhang, S. Y. Chou, Appl. Phys. Lett. 2003, 83, 1632.

[5] D. Pisignano, L. Persano, M. F. Raganato, P. Visconti, R. Cingolani, G. Barbarella, L. Favaretto, G. Gigli, Adv. Mater. 2004, 16, 525.

[6] M.-S. Kim, J.-S. Kim, J. Cho, M. Stein, L. J. Guo, J. Kim, Appl. Phys. Lett. 2007, 90, 123113.

[7] W. Wu, B. Cui, X. Sun, W. Zhang, L. Zhuang, L. Kong, S. Y. Chou, J. Vac. Sci. Technol. B 1998, 16, 3825.
[8] J. I. Martin, J. Nogues, K. Liu, J. L. Vicent, I. K. Schuller, J. Magn. Magn. Mater. 2003, 256, 449.

[9] H. Cao, Z. N. Yu, J. Wang, J. O. Tegenfeldt, R. H. Austin, E. Chen, W. Wu, S. Y. Chou, Appl. Phys. Lett. 2002, 81, 174.

[10] L. J. Guo, X. Cheng, C. F. Chou, Nano Lett. 2004, 4, 69.

[11] D. Falconnet, D. Pasqui, S. Park, R. Eckert, H. Schift, J. Gobrecht, R. Barbucci, M. Textor, Nano Lett. 2004, 4, 1909.

[12] J. D. Hoff, L. J. Cheng, E. Meyhofer, L. J. Guo, A. J. Hunt, Nano Lett. 2004, 4, 853.

[13] H. Tan, A. Gilbertson, S. Y. Chou, J. Vac. Sci. Technol. B 1998, 16 , 3926.

[14] L.-R. Bao, X. Cheng, X. D. Huang, L. J. Guo, S. W. Pang, A. F. Yee, J. Vac. Sci. Technol. B 2002, 20, 2881.

[15] J. Zaumseil, M. A. Meitl, J. W. P. Hsu, B. R. Acharya, K. W. Baldwin Y.-L. Loo, J. A. Rogers, Nano Lett. 2003, 3, 1223.

[16] D. Suh, S.-J. Choi, H. H. Lee, Adv. Mater. 2005, 17, 1554.

[17] G. B. Onoa, T. B. O'Reilly, M. E. Walsh, H. I. Smith, Nanotechnol. 2005, 12, 2799.

[18] S. Ahn, K. Lee, J. Kim, S. H. Kim, J. Park, S. H. Lee, P. Yoon, Microelectron. Eng. 2005, 78-79, 314.

[19] C. P.-Hernandez, J.-S. Kim, L. J. Guo, P.-F. Fu, Adv. Mater. 2007, 19 , 1222.

[20] X. Cheng, L. J. Guo, P.-F. Fu, Adv. Mater. 2005, 17, 1419.

[21] M. Colburn, S. C. Johnson, M. D. Stewart, S. Damle, T. C. Bailey, B. Choi, M. Wedlake, T. B. Michaelson, S. V. Sreenivasan, J. G. Ekerdt, C. G. Willson, Proc. SPIE-Int. Soc. Opt. Eng. 1999, 3676, 379.

[22] Y. Ekinci, H. H. Solak, C. David, H. Sigg, Opt. Express 2006, 14, 2323.

[23] S. H. Ahn, J.-S. Kim, L. J. Guo, J. Vac. Sci. Technol. B 2007, 25, 2388. 